\title{
Difference and Difference Quotient. Part II
}

\author{
Bo Li \\ Qingdao University of Science \\ and Technology \\ China \\ Yanping Zhuang \\ and Technology \\ China \\ Xiquan Liang \\ Qingdao University of Science \\ and Technology \\ China
}

\begin{abstract}
Summary. In this article, we give some important properties of forward difference, backward difference, central difference and difference quotient and forward difference, backward difference, central difference and difference quotient formulas of some special functions [11].
\end{abstract}

MML identifier: $\underline{\mathrm{DIFF}_{-} 2}$, version: $\underline{7.8 .094 .97 .1001}$

The articles [8], [1], [4], [2], [3], [5], [7], [12], [13], [6], [9], and [10] provide the notation and terminology for this paper.

We follow the rules: $h, r, r_{1}, r_{2}, x_{0}, x_{1}, x_{2}, x_{3}, x_{4}, x_{5}, x, a, b, c, k$ denote real numbers and $f, f_{1}, f_{2}$ denote functions from $\mathbb{R}$ into $\mathbb{R}$.

Next we state a number of propositions:

$(1)^{1} \Delta[f](x, x+h)=\frac{\left(\vec{\Delta}_{h}[f]\right)(1)(x)}{h}$.

(2) If $h \neq 0$, then $\Delta[f](x, x+h, x+2 \cdot h)=\frac{\left(\vec{\Delta}_{h}[f]\right)(2)(x)}{2 \cdot h^{2}}$.

(3) $\Delta[f](x-h, x)=\frac{\left(\vec{\nabla}_{h}[f]\right)(1)(x)}{h}$.

(4) If $h \neq 0$, then $\Delta[f](x-2 \cdot h, x-h, x)=\frac{\left(\vec{\nabla}_{h}[f]\right)(2)(x)}{2 \cdot h^{2}}$.

(5) $\Delta[r f]\left(x_{0}, x_{1}, x_{2}\right)=r \cdot \Delta[f]\left(x_{0}, x_{1}, x_{2}\right)$.

(6) $\Delta\left[f_{1}+f_{2}\right]\left(x_{0}, x_{1}, x_{2}\right)=\Delta\left[f_{1}\right]\left(x_{0}, x_{1}, x_{2}\right)+\Delta\left[f_{2}\right]\left(x_{0}, x_{1}, x_{2}\right)$.

${ }^{1}$ The notation $\Delta(f, x, y)$ has been changed to $\Delta[f](x, y)$. More in Addenda. 
(7) $\Delta\left[r_{1} f_{1}+r_{2} f_{2}\right]\left(x_{0}, x_{1}, x_{2}\right)=r_{1} \cdot \Delta\left[f_{1}\right]\left(x_{0}, x_{1}, x_{2}\right)+r_{2} \cdot \Delta\left[f_{2}\right]\left(x_{0}, x_{1}, x_{2}\right)$.

(8) $\Delta[r f]\left(x_{0}, x_{1}, x_{2}, x_{3}\right)=r \cdot \Delta[f]\left(x_{0}, x_{1}, x_{2}, x_{3}\right)$.

(9) $\Delta\left[f_{1}+f_{2}\right]\left(x_{0}, x_{1}, x_{2}, x_{3}\right)=\Delta\left[f_{1}\right]\left(x_{0}, x_{1}, x_{2}, x_{3}\right)+\Delta\left[f_{2}\right]\left(x_{0}, x_{1}, x_{2}, x_{3}\right)$.

(10) $\Delta\left[r_{1} f_{1}+r_{2} f_{2}\right]\left(x_{0}, x_{1}, x_{2}, x_{3}\right)=r_{1} \cdot \Delta\left[f_{1}\right]\left(x_{0}, x_{1}, x_{2}, x_{3}\right)+r_{2} \cdot$ $\Delta\left[f_{2}\right]\left(x_{0}, x_{1}, x_{2}, x_{3}\right)$.

Let $f$ be a real-yielding function and let $x_{0}, x_{1}, x_{2}, x_{3}, x_{4}$ be real numbers. The functor $\Delta[f]\left(x_{0}, x_{1}, x_{2}, x_{3}, x_{4}\right)$ yielding a real number is defined as follows: (Def. 1$) \quad \Delta[f]\left(x_{0}, x_{1}, x_{2}, x_{3}, x_{4}\right)=\frac{\Delta[f]\left(x_{0}, x_{1}, x_{2}, x_{3}\right)-\Delta[f]\left(x_{1}, x_{2}, x_{3}, x_{4}\right)}{x_{0}-x_{4}}$.

Next we state three propositions:

(11) $\Delta[r f]\left(x_{0}, x_{1}, x_{2}, x_{3}, x_{4}\right)=r \cdot \Delta[f]\left(x_{0}, x_{1}, x_{2}, x_{3}, x_{4}\right)$.

(12) $\Delta\left[f_{1}+f_{2}\right]\left(x_{0}, x_{1}, x_{2}, x_{3}, x_{4}\right)=\Delta\left[f_{1}\right]\left(x_{0}, x_{1}, x_{2}, x_{3}, x_{4}\right)+\Delta\left[f_{2}\right]\left(x_{0}, x_{1}, x_{2}\right.$, $\left.x_{3}, x_{4}\right)$.

(13) $\Delta\left[r_{1} f_{1}+r_{2} f_{2}\right]\left(x_{0}, x_{1}, x_{2}, x_{3}, x_{4}\right)=r_{1} \cdot \Delta\left[f_{1}\right]\left(x_{0}, x_{1}, x_{2}, x_{3}, x_{4}\right)+r_{2} \cdot$ $\Delta\left[f_{2}\right]\left(x_{0}, x_{1}, x_{2}, x_{3}, x_{4}\right)$.

Let $f$ be a real-yielding function and let $x_{0}, x_{1}, x_{2}, x_{3}, x_{4}, x_{5}$ be real numbers. The functor $\Delta[f]\left(x_{0}, x_{1}, x_{2}, x_{3}, x_{4}, x_{5}\right)$ yields a real number and is defined as follows:

(Def. 2) $\Delta[f]\left(x_{0}, x_{1}, x_{2}, x_{3}, x_{4}, x_{5}\right)=\frac{\Delta[f]\left(x_{0}, x_{1}, x_{2}, x_{3}, x_{4}\right)-\Delta[f]\left(x_{1}, x_{2}, x_{3}, x_{4}, x_{5}\right)}{x_{0}-x_{5}}$.

We now state a number of propositions:

(14) $\Delta[r f]\left(x_{0}, x_{1}, x_{2}, x_{3}, x_{4}, x_{5}\right)=r \cdot \Delta[f]\left(x_{0}, x_{1}, x_{2}, x_{3}, x_{4}, x_{5}\right)$.

(15) $\Delta\left[f_{1}+f_{2}\right]\left(x_{0}, x_{1}, x_{2}, x_{3}, x_{4}, x_{5}\right)=\Delta\left[f_{1}\right]\left(x_{0}, x_{1}, x_{2}, x_{3}, x_{4}, x_{5}\right)+\Delta\left[f_{2}\right]\left(x_{0}\right.$, $\left.x_{1}, x_{2}, x_{3}, x_{4}, x_{5}\right)$.

(16) $\Delta\left[r_{1} f_{1}+r_{2} f_{2}\right]\left(x_{0}, x_{1}, x_{2}, x_{3}, x_{4}, x_{5}\right)=r_{1} \cdot \Delta\left[f_{1}\right]\left(x_{0}, x_{1}, x_{2}, x_{3}, x_{4}, x_{5}\right)+$ $r_{2} \cdot \Delta\left[f_{2}\right]\left(x_{0}, x_{1}, x_{2}, x_{3}, x_{4}, x_{5}\right)$.

(17) If $x_{0}, x_{1}, x_{2}$ are mutually different, then $\Delta[f]\left(x_{0}, x_{1}, x_{2}\right)=$ $\frac{f\left(x_{0}\right)}{\left(x_{0}-x_{1}\right) \cdot\left(x_{0}-x_{2}\right)}+\frac{f\left(x_{1}\right)}{\left(x_{1}-x_{0}\right) \cdot\left(x_{1}-x_{2}\right)}+\frac{f\left(x_{2}\right)}{\left(x_{2}-x_{0}\right) \cdot\left(x_{2}-x_{1}\right)}$.

(18) If $x_{0}, x_{1}, x_{2}, x_{3}$ are mutually different, then $\Delta[f]\left(x_{0}, x_{1}, x_{2}, x_{3}\right)=$ $\Delta[f]\left(x_{1}, x_{2}, x_{3}, x_{0}\right)$ and $\Delta[f]\left(x_{0}, x_{1}, x_{2}, x_{3}\right)=\Delta[f]\left(x_{3}, x_{2}, x_{1}, x_{0}\right)$.

(19) If $x_{0}, x_{1}, x_{2}, x_{3}$ are mutually different, then $\Delta[f]\left(x_{0}, x_{1}, x_{2}, x_{3}\right)=$ $\Delta[f]\left(x_{1}, x_{0}, x_{2}, x_{3}\right)$ and $\Delta[f]\left(x_{0}, x_{1}, x_{2}, x_{3}\right)=\Delta[f]\left(x_{1}, x_{2}, x_{0}, x_{3}\right)$.

(20) If $f$ is constant, then $\Delta[f]\left(x_{0}, x_{1}, x_{2}\right)=0$.

(21) If $x_{0} \neq x_{1}$, then $\Delta[a \square+b]\left(x_{0}, x_{1}\right)=a$.

(22) If $x_{0}, x_{1}, x_{2}$ are mutually different, then $\Delta[a \square+b]\left(x_{0}, x_{1}, x_{2}\right)=0$.

(23) If $x_{0}, x_{1}, x_{2}, x_{3}$ are mutually different, then $\Delta[a \square+b]\left(x_{0}, x_{1}, x_{2}, x_{3}\right)=0$.

(24) For every $x$ holds $\left(\Delta_{h}[a \square+b]\right)(x)=a \cdot h$.

(25) For every $x$ holds $\left(\nabla_{h}[a \square+b]\right)(x)=a \cdot h$.

(26) For every $x$ holds $\left(\delta_{h}[a \square+b]\right)(x)=a \cdot h$. 
(27) If for every $x$ holds $f(x)=a \cdot x^{2}+b \cdot x+c$ and $x_{0} \neq x_{1}$, then $\Delta[f]\left(x_{0}, x_{1}\right)=$ $a \cdot\left(x_{0}+x_{1}\right)+b$.

(28) If for every $x$ holds $f(x)=a \cdot x^{2}+b \cdot x+c$ and $x_{0}, x_{1}, x_{2}$ are mutually different, then $\Delta[f]\left(x_{0}, x_{1}, x_{2}\right)=a$.

(29) If for every $x$ holds $f(x)=a \cdot x^{2}+b \cdot x+c$ and $x_{0}, x_{1}, x_{2}, x_{3}$ are mutually different, then $\Delta[f]\left(x_{0}, x_{1}, x_{2}, x_{3}\right)=0$.

(30) If for every $x$ holds $f(x)=a \cdot x^{2}+b \cdot x+c$ and $x_{0}, x_{1}, x_{2}, x_{3}, x_{4}$ are mutually different, then $\Delta[f]\left(x_{0}, x_{1}, x_{2}, x_{3}, x_{4}\right)=0$.

(31) If for every $x$ holds $f(x)=a \cdot x^{2}+b \cdot x+c$, then for every $x$ holds $\left(\Delta_{h}[f]\right)(x)=2 \cdot a \cdot h \cdot x+a \cdot h^{2}+b \cdot h$.

(32) If for every $x$ holds $f(x)=a \cdot x^{2}+b \cdot x+c$, then for every $x$ holds $\left(\nabla_{h}[f]\right)(x)=\left(2 \cdot a \cdot h \cdot x-a \cdot h^{2}\right)+b \cdot h$.

(33) If for every $x$ holds $f(x)=a \cdot x^{2}+b \cdot x+c$, then for every $x$ holds $\left(\delta_{h}[f]\right)(x)=2 \cdot a \cdot h \cdot x+b \cdot h$.

(34) If for every $x$ holds $f(x)=\frac{k}{x}$ and $x_{0} \neq x_{1}$ and $x_{0} \neq 0$ and $x_{1} \neq 0$, then $\Delta[f]\left(x_{0}, x_{1}\right)=-\frac{k}{x_{0} \cdot x_{1}}$.

(35) If for every $x$ holds $f(x)=\frac{k}{x}$ and $x_{0} \neq 0$ and $x_{1} \neq 0$ and $x_{2} \neq 0$ and $x_{0}$, $x_{1}, x_{2}$ are mutually different, then $\Delta[f]\left(x_{0}, x_{1}, x_{2}\right)=\frac{k}{x_{0} \cdot x_{1} \cdot x_{2}}$.

(36) Suppose for every $x$ holds $f(x)=\frac{k}{x}$ and $x_{0} \neq 0$ and $x_{1} \neq 0$ and $x_{2} \neq 0$ and $x_{3} \neq 0$ and $x_{0}, x_{1}, x_{2}, x_{3}$ are mutually different. Then $\Delta[f]\left(x_{0}, x_{1}, x_{2}, x_{3}\right)=-\frac{k}{x_{0} \cdot x_{1} \cdot x_{2} \cdot x_{3}}$.

(37) Suppose for every $x$ holds $f(x)=\frac{k}{x}$ and $x_{0} \neq 0$ and $x_{1} \neq 0$ and $x_{2} \neq 0$ and $x_{3} \neq 0$ and $x_{4} \neq 0$ and $x_{0}, x_{1}, x_{2}, x_{3}, x_{4}$ are mutually different. Then $\Delta[f]\left(x_{0}, x_{1}, x_{2}, x_{3}, x_{4}\right)=\frac{k}{x_{0} \cdot x_{1} \cdot x_{2} \cdot x_{3} \cdot x_{4}}$.

(38) If for every $x$ holds $f(x)=\frac{k}{x}$ and $x \neq 0$ and $x+h \neq 0$, then for every $x$ holds $\left(\Delta_{h}[f]\right)(x)=\frac{-k \cdot h}{(x+h) \cdot x}$.

(39) If for every $x$ holds $f(x)=\frac{k}{x}$ and $x \neq 0$ and $x-h \neq 0$, then for every $x$ holds $\left(\nabla_{h}[f]\right)(x)=\frac{-k \cdot h}{(x-h) \cdot x}$.

(40) If for every $x$ holds $f(x)=\frac{k}{x}$ and $x+\frac{h}{2} \neq 0$ and $x-\frac{h}{2} \neq 0$, then for every $x$ holds $\left(\delta_{h}[f]\right)(x)=\frac{-k \cdot h}{\left(x-\frac{h}{2}\right) \cdot\left(x+\frac{h}{2}\right)}$.

(41) $\Delta$ [the function $\sin ]\left(x_{0}, x_{1}\right)=\frac{2 \cdot \cos \left(\frac{x_{0}+x_{1}}{2}\right) \cdot \sin \left(\frac{x_{0}-x_{1}}{2}\right)}{x_{0}-x_{1}}$.

(42) For every $x$ holds $\left(\Delta_{h}\right.$ [the function $\left.\left.\sin \right]\right)(x)=2 \cdot\left(\cos \left(\frac{2 \cdot x+h}{2}\right) \cdot \sin \left(\frac{h}{2}\right)\right)$.

(43) For every $x$ holds $\left(\nabla_{h}\right.$ [the function $\left.\left.\sin \right]\right)(x)=2 \cdot\left(\cos \left(\frac{2 \cdot x-h}{2}\right) \cdot \sin \left(\frac{h}{2}\right)\right)$.

(44) For every $x$ holds $\left(\delta_{h}[\right.$ the function $\left.\sin ]\right)(x)=2 \cdot\left(\cos x \cdot \sin \left(\frac{h}{2}\right)\right)$.

(45) $\Delta$ [the function $\cos ]\left(x_{0}, x_{1}\right)=-\frac{2 \cdot \sin \left(\frac{x_{0}+x_{1}}{2}\right) \cdot \sin \left(\frac{x_{0}-x_{1}}{2}\right)}{x_{0}-x_{1}}$.

(46) For every $x$ holds $\left(\Delta_{h}\right.$ [the function $\left.\left.\cos \right]\right)(x)=-2 \cdot\left(\sin \left(\frac{2 \cdot x+h}{2}\right) \cdot \sin \left(\frac{h}{2}\right)\right)$.

(47) For every $x$ holds $\left(\nabla_{h}[\right.$ the function $\left.\cos ]\right)(x)=-2 \cdot\left(\sin \left(\frac{2 \cdot x-h}{2}\right) \cdot \sin \left(\frac{h}{2}\right)\right)$. 
(48) For every $x$ holds $\left(\delta_{h}[\right.$ the function $\left.\cos ]\right)(x)=-2 \cdot\left(\sin x \cdot \sin \left(\frac{h}{2}\right)\right)$.

(49) $\Delta[$ (the function sin) (the function $\sin )]\left(x_{0}, x_{1}\right)=\frac{\frac{1}{2} \cdot\left(\cos \left(2 \cdot x_{1}\right)-\cos \left(2 \cdot x_{0}\right)\right)}{x_{0}-x_{1}}$.

(50) For every $x$ holds $\left(\Delta_{h}[(\right.$ the function sin) (the function $\left.\sin )]\right)(x)=\frac{1}{2}$. $(\cos (2 \cdot x)-\cos (2 \cdot(x+h)))$.

(51) For every $x$ holds $\left(\nabla_{h}[\right.$ (the function sin) (the function $\left.\left.\sin )\right]\right)(x)=\frac{1}{2}$. $(\cos (2 \cdot(x-h))-\cos (2 \cdot x))$.

(52) For every $x$ holds $\left(\delta_{h}[(\right.$ the function $\sin )$ (the function sin) $\left.]\right)(x)=\frac{1}{2}$. $(\cos (2 \cdot x-h)-\cos (2 \cdot x+h))$.

(53) $\Delta[$ (the function $\sin$ ) (the function $\cos )]\left(x_{0}, x_{1}\right)=\frac{\frac{1}{2} \cdot\left(\sin \left(2 \cdot x_{0}\right)-\sin \left(2 \cdot x_{1}\right)\right)}{x_{0}-x_{1}}$.

(54) For every $x$ holds $\left(\Delta_{h}[\right.$ (the function sin) (the function $\left.\left.\cos )\right]\right)(x)=\frac{1}{2}$. $(\sin (2 \cdot(x+h))-\sin (2 \cdot x))$.

(55) For every $x$ holds $\left(\nabla_{h}[\right.$ (the function $\sin )$ (the function $\left.\left.\left.\cos \right)\right]\right)(x)=\frac{1}{2}$. $(\sin (2 \cdot x)-\sin (2 \cdot(x-h)))$.

(56) For every $x$ holds $\left(\delta_{h}[(\right.$ the function sin) $($ the function $\cos )])(x)=\frac{1}{2}$. $(\sin (2 \cdot x+h)-\sin (2 \cdot x-h))$.

(57) $\Delta[($ the function cos) (the function $\cos )]\left(x_{0}, x_{1}\right)=\frac{\frac{1}{2} \cdot\left(\cos \left(2 \cdot x_{0}\right)-\cos \left(2 \cdot x_{1}\right)\right)}{x_{0}-x_{1}}$.

(58) For every $x$ holds $\left(\Delta_{h}[\right.$ (the function cos) (the function $\left.\left.\cos )\right]\right)(x)=\frac{1}{2}$. $(\cos (2 \cdot(x+h))-\cos (2 \cdot x))$.

(59) For every $x$ holds $\left(\nabla_{h}[\right.$ (the function cos) (the function cos) $\left.]\right)(x)=\frac{1}{2}$. $(\cos (2 \cdot x)-\cos (2 \cdot(x-h)))$.

(60) For every $x$ holds $\left(\delta_{h}[(\right.$ the function $\cos )($ the function $\left.\cos )]\right)(x)=\frac{1}{2}$. $(\cos (2 \cdot x+h)-\cos (2 \cdot x-h))$.

(61) $\Delta[$ (the function $\sin )$ (the function $\sin$ ) (the function $\cos )]\left(x_{0}, x_{1}\right)=$ $-\frac{\frac{1}{2} \cdot\left(\sin \left(\frac{3 \cdot\left(x_{1}+x_{0}\right)}{2}\right) \cdot \sin \left(\frac{3 \cdot\left(x_{1}-x_{0}\right)}{2}\right)+\sin \left(\frac{x_{0}+x_{1}}{2}\right) \cdot \sin \left(\frac{x_{0}-x_{1}}{2}\right)\right)}{x_{0}-x_{1}}$.

(62) Let given $x$. Then $\left(\Delta_{h}[\right.$ (the function $\sin )$ (the function $\left.\sin \right)$ (the function $\cos )])(x)=\frac{1}{2} \cdot\left(\sin \left(\frac{6 \cdot x+3 \cdot h}{2}\right) \cdot \sin \left(\frac{3 \cdot h}{2}\right)-\sin \left(\frac{2 \cdot x+h}{2}\right) \cdot \sin \left(\frac{h}{2}\right)\right)$.

(63) Let given $x$. Then $\left(\nabla_{h}[(\right.$ the function $\sin )$ (the function $\sin )$ (the function $\cos )])(x)=\frac{1}{2} \cdot\left(\sin \left(\frac{6 \cdot x-3 \cdot h}{2}\right) \cdot \sin \left(\frac{3 \cdot h}{2}\right)\right)-\frac{1}{2} \cdot\left(\sin \left(\frac{2 \cdot x-h}{2}\right) \cdot \sin \left(\frac{h}{2}\right)\right)$.

(64) For every $x$ holds $\left(\delta_{h}[(\right.$ the function $\sin )$ (the function $\sin )$ (the function $\cos )])(x)=-\frac{1}{2} \cdot\left(\sin x \cdot \sin \left(\frac{h}{2}\right)\right)+\frac{1}{2} \cdot\left(\sin (3 \cdot x) \cdot \sin \left(\frac{3 \cdot h}{2}\right)\right)$.

(65) $\Delta[$ (the function $\sin )$ (the function $\cos$ ) (the function $\cos )]\left(x_{0}, x_{1}\right)=$ $\frac{\frac{1}{2} \cdot\left(\cos \left(\frac{x_{0}+x_{1}}{2}\right) \cdot \sin \left(\frac{x_{0}-x_{1}}{2}\right)+\cos \left(\frac{3 \cdot\left(x_{0}+x_{1}\right)}{2}\right) \cdot \sin \left(\frac{3 \cdot\left(x_{0}-x_{1}\right)}{2}\right)\right)}{x_{0}-x_{1}}$.

(66) Let given $x$. Then $\left(\Delta_{h}[\right.$ (the function $\sin )$ (the function $\left.\cos \right)$ (the function $\cos )])(x)=\frac{1}{2} \cdot\left(\cos \left(\frac{2 \cdot x+h}{2}\right) \cdot \sin \left(\frac{h}{2}\right)+\cos \left(\frac{6 \cdot x+3 \cdot h}{2}\right) \cdot \sin \left(\frac{3 \cdot h}{2}\right)\right)$.

(67) Let given $x$. Then $\left(\nabla_{h}[(\right.$ the function $\sin )$ (the function $\cos )$ (the function $\cos )])(x)=\frac{1}{2} \cdot\left(\cos \left(\frac{2 \cdot x-h}{2}\right) \cdot \sin \left(\frac{h}{2}\right)+\cos \left(\frac{6 \cdot x-3 \cdot h}{2}\right) \cdot \sin \left(\frac{3 \cdot h}{2}\right)\right)$. 
(68) For every $x$ holds $\left(\delta_{h}[(\right.$ the function $\sin )$ (the function cos) (the function $\cos )])(x)=\frac{1}{2} \cdot\left(\cos x \cdot \sin \left(\frac{h}{2}\right)+\cos (3 \cdot x) \cdot \sin \left(\frac{3 \cdot h}{2}\right)\right)$.

(69) If $x_{0} \in \operatorname{dom}\left(\right.$ the function tan) and $x_{1} \in \operatorname{dom}$ (the function tan), then $\Delta[$ the function $\tan ]\left(x_{0}, x_{1}\right)=\frac{\sin \left(x_{0}-x_{1}\right)}{\cos x_{0} \cdot \cos x_{1} \cdot\left(x_{0}-x_{1}\right)}$.

(70) If $x_{0} \in \operatorname{dom}\left(\right.$ the function cot) and $x_{1} \in \operatorname{dom}$ (the function cot), then $\Delta[$ the function $\cot ]\left(x_{0}, x_{1}\right)=-\frac{\sin \left(x_{0}-x_{1}\right)}{\sin x_{0} \cdot \sin x_{1} \cdot\left(x_{0}-x_{1}\right)}$.

(71) Suppose $x_{0} \in$ dom (the function cosec) and $x_{1} \in$ dom (the function cosec). Then $\Delta[$ the function $\operatorname{cosec}]\left(x_{0}, x_{1}\right)=\frac{2 \cdot \cos \left(\frac{x_{1}+x_{0}}{2}\right) \cdot \sin \left(\frac{x_{1}-x_{0}}{2}\right)}{\sin x_{1} \cdot \sin x_{0} \cdot\left(x_{0}-x_{1}\right)}$.

(72) Suppose $x_{0} \in \operatorname{dom}$ (the function sec) and $x_{1} \in \operatorname{dom}$ (the function sec). Then $\Delta[$ the function sec $]\left(x_{0}, x_{1}\right)=-\frac{2 \cdot \sin \left(\frac{x_{1}+x_{0}}{2}\right) \cdot \sin \left(\frac{x_{1}-x_{0}}{2}\right)}{\cos x_{1} \cdot \cos x_{0} \cdot\left(x_{0}-x_{1}\right)}$.

\section{REFERENCES}

[1] Grzegorz Bancerek. The ordinal numbers. Formalized Mathematics, 1(1):91-96, 1990.

[2] Czesław Byliński. Functions and their basic properties. Formalized Mathematics, 1(1):55$65,1990$.

[3] Czesław Byliński. Functions from a set to a set. Formalized Mathematics, 1(1):153-164, 1990.

[4] Czesław Byliński. Some basic properties of sets. Formalized Mathematics, 1(1):47-53, 1990.

[5] Jarosław Kotowicz. Real sequences and basic operations on them. Formalized Mathematics, 1(2):269-272, 1990.

[6] Bo Li, Yan Zhang, and Xiquan Liang. Difference and difference quotient. Formalized Mathematics, 14(3):115-119, 2006.

[7] Beata Perkowska. Functional sequence from a domain to a domain. Formalized Mathematics, 3(1):17-21, 1992.

[8] Andrzej Trybulec and Czesław Byliński. Some properties of real numbers. Formalized Mathematics, 1(3):445-449, 1990.

[9] Andrzej Trybulec and Yatsuka Nakamura. On the decomposition of a simple closed curve into two arcs. Formalized Mathematics, 10(3):163-167, 2002.

[10] Peng Wang and Bo Li. Several differentiation formulas of special functions. Part V. Formalized Mathematics, 15(3):73-79, 2007.

[11] Renhong Wang. Numerical approximation. Higher Education Press, Beijing, 1999.

[12] Edmund Woronowicz. Relations defined on sets. Formalized Mathematics, 1(1):181-186, 1990.

[13] Yuguang Yang and Yasunari Shidama. Trigonometric functions and existence of circle ratio. Formalized Mathematics, 7(2):255-263, 1998. 